\title{
ZERO-INFLATED ENDOGENOUS COUNT IN CENSORED MODEL: EFFECTS OF INFORMAL FAMILY CARE ON FORMAL HEALTH CARE
}

\author{
MYOUNG-JAE LEE ${ }^{\mathrm{a}, \mathrm{b}, *}$ and YOUNG-SOOK KIM ${ }^{\mathrm{c}}$ \\ ${ }^{a}$ Department of Economics, Korea University, Seoul, South Korea \\ ${ }^{\mathrm{b}}$ Research School of Economics, Australian National University, Canberra, Australia \\ ${ }^{\mathrm{c}}$ Korean Women's Development Institute, Seoul, South Korea
}

SUMMARY

\begin{abstract}
If informal family health care is a substitute for formal health care, then there is a scope to reduce formal health care cost by promoting informal family health care. With the use of Korean data for the elderly, this paper estimates the effects of informal family health care on formal health care, where the former is measured by the number of caregivers and the latter is measured by the formal health care expenditure. This task, however, poses a number of difficulties. The first is that the number of the family caregivers is an endogenous count regressor. The second is that there are too many zeros in the count (85\%). The third is that the response variable also has a nontrivial proportion of zeros (14\%). This paper overcomes these problems by combining 'control function approach', 'zero-inflated' counts, and a semiparametric estimator for censored models. The resulting procedure avoids strong parametric assumptions and behaves well computationally. Our main empirical finding is that informal family health care has a large substitute effect for diabetics and that there are also weak evidences that informal family health care has substitute effects for high blood pressure and mental diseases. Copyright () 2012 John Wiley \& Sons, Ltd.
\end{abstract}

Received 20 September 2011; Revised 12 January 2012; Accepted 26 March 2012

KEY WORDS: informal health care; formal health care; count variable; control function; zero-inflated Poisson; censored model

\section{INTRODUCTION}

With low fertility rates prevailing in most developed countries, the populations age fast, and this entails a high demand for health care. If the health care cost is borne only by formal health care, then eventually there may be a point at which the health care system ceases to be sustainable. If formal health care can be replaced to some extent by informal family health care, then this may lead to a considerable reduction on the formal health care cost.

In the literature of health economics, there are studies that examined the effects of informal health care on formal health care, which often find that informal care substitutes for formal care. Although there are studies such as Charles and Sevak (2005) showing that informal care (measured by the dummy for any informal care) is a substitute for nursing home care (measured by the dummy for ever staying in nursing home), in the following, we briefly review three studies that are the most relevant to our paper: Van Houtven and Norton (2004), Bolin et al. (2008), and Bonsang (2009).

In Van Houtven and Norton (2004), informal care is the care hours provided by all children (their spouse and their children), and formal cares including nursing home care and outpatient care are of eight different types in total (mostly continuously distributed, but formal home care and outpatient surgery are binary). Only about $19 \%$ of the respondents received informal care. Van Houtven and Norton used US data: 1998 Health and Retirement Survey (HRS) and 1995 Asset and Health Dynamics Among the Oldest-Old Panel Survey (AHEAD). Van Houtven and Norton found that informal care is mostly a substitute except for outpatient surgery.

*Correspondence to: Department of Economics, Korea University, Anam-dong, Sungbuk-gu, Seoul 136-701, South Korea. E-mail: myoungjae@korea.ac.kr 
In Bolin et al. (2008), nine formal care variables are used including formal home care, visits to doctors and hospitalization days. Their informal care (informal care hours from children and grandchildren) has the nonzero proportion ranging 19-40\% across the countries in their 2004 European data 'SHARE'. Bolin et al. found that informal care is a substitute for formal home care, but a complement to doctor and hospital visits, and that the effects vary depending on the region (i.e., informal care interacts with the region dummies).

In Bonsang (2009), informal care is the care hours provided by children of the respondent (a single-living elderly), and formal cares are paid domestic help (low skilled) and nursing care (high skilled); both formal cares are home cares. Using the 2004 European data SHARE, Bonsang found that informal care is a substitute for the low-skilled formal home care, but a weak complement for the high-skilled formal home care, and that the substitution effect decreases as the level of disability of the elderly person increases (i.e., informal care interacts with the disability level).

In terms of methods, Van Houtven and Norton (2004), Bolin et al. (2008), and Bonsang (2009) used a 'twopart approach'. But strictly speaking, the methods used there to deal with endogenous regressors apply only when the endogenous regressors are continuously distributed. Probably because of this restriction, least squares estimator (LSE) was used to estimate the reduced form (RF) model for informal care that is an endogenous regressor for formal care (the response variable). But the LSE is problematic because the informal care variable includes too many zeros. Also, the response variable has a nontrivial proportion of zeros. In short, both the main endogenous regressor of interest and the response variable are either discrete or mixed (discrete/continuous), and thus, linear models are not allowed.

One reason for the endogeneity of informal care is that both formal and informal cares may be determined simultaneously. Another reason is that both cares may share common factors-most notably, health status. But controlling for health status is problematic because it may be influenced by both cares. Note that, as instruments for informal care, distances to children, placement of daughters in the birth order, or the number of (female) children have been used in the literature.

Whereas there is no particularly good solution for the endogeneity problem, this paper will propose a two-stage procedure to overcome the problems of too many zeros in a nonnegative endogenous regressor (informal care) and a nontrivial proportion of zeros in the response variable (formal care). For the RF estimation of the nonnegative regressor, we will be using 'quasi-Poisson' approach. For too many zeros, we will be using the zero-inflated Poisson of Lambert (1992), which has been in fact preceded by Mullahy (1986) under the name 'with zero' approach. In a nutshell, our two-stage procedure is applicable to censored models with nonnegative endogenous regressors including count variables where the endogenous regressors have too many zeros.

The rest of this paper is organized as follows. Section 2 shows the details of the two-stage procedure. Section 3 applies the estimator to Korean data to estimate the effect of informal care on formal care, where informal care is the number of family caregivers (thus a count). Finally, Section 4 concludes. A word on notation before proceeding further: ' $a \coprod b \mid c$ ' denotes the independence between $a$ and $b$ given $c$.

\section{TWO-STAGE PROCEDURE}

\subsection{Model assumptions}

Suppose that $y_{1} \geq 0$ is formal care, $y_{2} \geq 0$ is informal care (a count), $x_{1}$ is a $k_{1} \times 1$ exogenous regressor vector for the $y_{1}$ structural form (SF) equation, and $x$ is the $k \times 1$ system exogenous regressor vector for $\left(y_{1}, y_{2}\right)$ that strictly includes $x_{1}$. That is, only $x_{1}$ in $x$ affects $y_{1}$ 'directly', and $x$ is the collection of the exogenous regressors affecting either $y_{1}$ or $y_{2}$. Observed are

$$
\left(x_{i}, y_{1 i}, y_{2 i}\right), \quad i=1, \quad \ldots, \quad N, \quad \text { which are iid across } i .
$$

Our approach applies not only to a count but also to nonnegative $y_{2}$. In view of the iid assumption, we will often omit the subscript $i$. 
Assume that the observed $y_{1}$ and $y_{2}$ are generated from its latent versions $y_{1}^{*}$ and $y_{2}^{*}$ as follows: for unknown parameters $\gamma_{y}, \gamma_{x}, \alpha$ and $\beta$, an error term $u_{i}$ and a binary variable $q_{i}$,

$$
\begin{aligned}
& \text { Censored SF } y_{1}: y_{1 i}=\max \left(0, y_{1 i}^{*}\right), \quad y_{1 i}^{*}=\gamma_{y} y_{2 i}+x_{1 i}^{\prime} \gamma_{x}+u_{i}, \quad u \mid x \text { symmetric; } \\
& \text { Selection RF } y_{2}: y_{2 i}=q_{i} y_{2 i}^{*}, \quad P\left(q=1 \mid x_{i}\right)=\frac{\exp \left(x_{i}^{\prime} \alpha\right)}{1+\exp \left(x_{i}^{\prime} \alpha\right)}, E\left(y_{2}^{*} \mid q=1, x_{i}\right)=\exp \left(x_{i}^{\prime} \beta\right) .
\end{aligned}
$$

Here, $y_{1}^{*}$ is modeled as left censored at zero with its error term $u$ given $x$ being symmetric around zero. This symmetry assumption is to use the symmetrically censored least squares estimator (SCL) of Powell (1986) and may be replaced by another semiparametric assumption if a different semiparametric estimator as in Powell (1984) or Lee (1992) is used.

The left-censored model for $y_{1}$ is different from the usual right-censored model in duration analysis where the latent duration exists but only its right-censored version is observed. Rather, the left-censored model comes from the 'corner-solution' nature where $y_{1}$ is a choice variable subject to nonnegativity whereas $y_{1}^{*}$ would be the choice variable without the constraint. Such a corner-solution model is a special case of a sample selection model that allows a model for zero or positive to differ from the model governing the positive part. In this regard, it might be better to model $y_{1}$ as a sample selection model rather than as the censored model, but the censored model is adopted for simplicity because dealing with a sample selection model is difficult — this would not matter much though as the proportion of zeros is low for $y_{1}$ in our data (14\%).

Because $x$ appears for $q$ and $y_{2}^{*}$, the $q$ and $y_{2}^{*}$ equations should be regarded as an $R F$. This RF view is necessary because $y_{1}$ does not appear for the $q$ and $y_{2}^{*}$ equations and also because $E\left(y_{2}^{*} \mid q=1, x\right)=\exp \left(x^{\prime} \beta\right)$ is adopted, not the more 'structural' $E\left(y_{2}^{*} \mid x\right)=\exp \left(x^{\prime} \beta\right)$. There are two views on RFs as noted in Lee (2012a). One view is that there is an SF for $y_{2}^{*}$ with $y_{1}$ and ' $x_{2}$ ' as the regressors, and substituting the $y_{1}$ SF and then solving the equation for $y_{2}^{*}$ yields the $y_{2}^{*} \mathrm{RF}$ with $x$ on the right hand side. The problem with this view is that it is not clear whether the equation is solvable for $y_{2}^{*}$ and if so, whether the solution is unique (and stable). The other view on RF is to take $E\left(y_{2}^{*} \mid x\right)$ as the $y_{2}^{*} \mathrm{RF}$ and use a parametric function for $E\left(y_{2}^{*} \mid x\right)$ as an approximation if desired. The problem with this view is that no information/structure can be imposed on $E\left(y_{2}^{*} \mid x\right)$, and the parametric form may be ad hoc.

Some further remarks about (2.1) are in order. First, a sample selection model holds for $y_{2}^{*}$ because $y_{2}^{*}$ is observed only when $q=1$; the binary 'selection variable' $q$ is assumed to follow the logit model, whereas $y_{2}^{*}$ given $q=1$ is posited to have an exponential regression function. Second, the key implication of the selection model for $y_{2}$ is

$$
E\left(y_{2} \mid x\right)=P(q=1 \mid x) E\left(y_{2}^{*} \mid q=1, x\right)=\frac{\exp \left(x^{\prime} \alpha\right)}{1+\exp \left(x^{\prime} \alpha\right)} \exp \left(x^{\prime} \beta\right) .
$$

Third, the expression 'too many zeros' may be formally defined as

$$
E\left[\left\{y_{2}-\frac{\exp \left(x^{\prime} \alpha\right)}{1+\exp \left(x^{\prime} \alpha\right)} \exp \left(x^{\prime} \beta\right)\right\}^{2}\right]<E\left[\left\{y_{2}-\exp \left(x^{\prime} \beta\right)\right\}^{2}\right]
$$

that is, the extra logit function providing a better prediction of $y_{2}$ than when only $\exp \left(x^{\prime} \beta\right)$ is used is defined as 'too many zeros in $y_{2}$ '. Because there exist different types of (confusing) sample selection models, we examine them closely in the following in relation to our selection model for $y_{2}$, drawing upon Lee (2012b).

Define $1[A]=1$ if $A$ holds and 0 otherwise, and call $y_{2}^{*}=0$ as the participation zero. It is helpful to compare three different models for $q$ in relation to the participation zero possibility:

Model1 : $q=1\left[y_{2}^{*}>0\right]$ where $y_{2}\left(=q y_{2}^{*}\right)=0$ implies $y_{2}^{*}=0 ;$

Model2 : $q$ determined by some variables $\left(\right.$ and $y_{2}^{*}$ ) with participation 0 possible;

Model3 : $q$ determined by some variables $\left(\right.$ and $\left.y_{2}^{*}\right)$ with participation 0 impossible. 
Model 1 is the corner solution model in which case $y_{2}$ also becomes a zero-censored model as $y_{1}$. Model 2 is relevant if $q=1$ is only an 'attempt/try' for an activity and $y_{2}^{*}$ is a 'performance' in the activity following the attempt/try. Model 3 is relevant if $q=1$ is having the actual activity and $y_{2}^{*}$ is the degree of the activity with zero ruled out.

For instance, $q=1$ may be an attempt/try to export, where $y_{2}^{*}=0$ is possible even if one tries $(q=1)$. Instead of an attempt/try, one may define $q=1$ as actually exporting and $y_{2}^{*}$ as the actual export volume that cannot be zero. Which one between Models 2 and 3 to adopt may depend on what is available in the data. If a variable $q$ for 'whether one desires to export or not' is available in the data along with the export volume including zero, then $y_{2}=q y_{2}^{*}$ is the observed export volume with $y_{2}^{*}=0$ possible. If only the actual export volume including zero is available, but not $q$, then one has no choice but to set $q=1\left[y_{2}>0\right]$ with participation zero ruled out. In our data, because there is no separate variable for $q$, we will set $q=1\left[y_{2}>0\right]$ to adopt Model 3 .

One may wonder 'why not adopt Model 1 for $y_{2}$ that looks simpler than Model 3' to do away with $\beta$ then the left-censored model at zero would hold for both $y_{1}$ and $y_{2}$. The answer is that there is really no difference between Models 1 and 3 for our empirical analysis, as $\beta$ is needed anyway. To see the point, suppose $y_{2}^{*}=x^{\prime} \alpha+v_{2}$ with $v_{2}$ being logistic independently of $x$ and Model 1 holds. Then,

$$
\begin{aligned}
& q=1\left[y_{2}^{*}>0\right]=1\left[x^{\prime} \alpha+v_{2}>0\right] \Rightarrow E(q \mid x)=\frac{\exp \left(x^{\prime} \alpha\right)}{1+\exp \left(x^{\prime} \alpha\right)} \text { and } \\
& E\left(y_{2}^{*} \mid q=1, x\right)=E\left(y_{2}^{*} \mid y_{2}^{*}>0, x\right)=x^{\prime} \alpha+E\left(v_{2} \mid v_{2}>-x^{\prime} \alpha, x\right) \neq \exp \left(x^{\prime} \alpha\right) .
\end{aligned}
$$

In this case, the exponential model is only an approximation for $x^{\prime} \alpha+E\left(v_{2} \mid v_{2}>-x^{\prime} \alpha, x\right)$, and consequently, we need to allow different parameters $\alpha$ for $E(q \mid x)$ and $\beta$ for $E\left(y_{2}^{*} \mid q=1, x\right)$ as when Model 3 is adopted.

\subsection{First stage to obtain control function}

In our two-stage procedure, the first stage consists of two parts: estimating $\alpha$ in the logit model for $E(q \mid x)$ and estimating $\beta$ in the exponential model for $E(y \mid q=1, x)=E\left(y_{2}^{*} \mid q=1, x\right)$. For the latter, one can use quasi-Poisson maximum likelihood estimator (QPOI) with the $q=1$ subsample: maximize the usual Poisson likelihood function with $q=1$ attached to use the 'sandwich-form' asymptotic variance. That is, the QPOI maximand for $b$ is

$$
\frac{1}{N} \sum_{i} q_{i}\left\{y_{2 i} x_{i}^{\prime} b-\exp \left(x_{i} b\right)\right\}
$$

and the asymptotic variance matrix is

$$
E^{-1}\left\{q x x^{\prime} \exp \left(x^{\prime} \beta\right)\right\} \cdot E\left[q x x^{\prime}\left\{y-\exp \left(x^{\prime} \beta\right)\right\}^{2}\right] \cdot E^{-1}\left\{q x x^{\prime} \exp \left(x^{\prime} \beta\right)\right\} .
$$

Denoting the first-stage estimators as $\hat{\alpha}$ and $\hat{\beta}$, the second stage is estimating $\gamma_{y}$ and $\gamma_{x}$ for the $y_{1}$ SF allowing for the endogeneity of $y_{2}$ in the $y_{1} \mathrm{SF}$. As reviewed in Lee (2012a), there are several different methods to deal with endogenous regressors in a limited dependent variable (LDV) model-the LDV model is the zerocensored model for $y_{1}$ in our case. Among those methods, the most convenient for our empirical analysis is 'control function (CF)' approach because many interaction terms between $y_{2}$ and elements of $x$ will be allowed. With the endogeneity of $y_{2}$ removed by a CF, we can freely allow such interaction terms, which would be complicated in the other approaches for the $y_{2}$ endogeneity.

Specifically, a residual $\hat{v}_{2}$ for $y_{2}$ is obtained from the first stage, and it is used as an extra regressor in the $y_{1} \mathrm{SF}$. Not just $\hat{v}_{2}$ but also $\hat{v}_{2}^{2}$ can be used if including it better removes the $y_{2}$ endogeneity. Then, $\left(\hat{v}_{2}, \hat{v}_{2}^{2}\right)$ becomes the $\mathrm{CF}$, and the $y_{2}$ endogeneity can be tested by looking at whether their coefficients are all zero or not. Going further from $\left(\hat{v}_{2}, \hat{v}_{2}^{2}\right)$, even higher-order terms of $\hat{v}_{2}$ or interaction terms between $\left(\hat{v}_{2}, \hat{v}_{2}^{2}\right)$ and $x$ may be used as well. The usual practice of using only $\hat{v}_{2}$ as a CF originates from the bivariate normality of 
$\left(u, v_{2}\right)$ with $E\left(u \mid v_{2}, x\right)=E\left(u \mid v_{2}\right)$ being a linear function of $v_{2}$. But in a semiparametric context, $E\left(u \mid v_{2}\right)$ may be nonlinear in $v_{2}$ (e.g., $E\left(u \mid v_{2}\right)=\rho_{1} v_{2}+\rho_{2} v_{2}^{2}$ with $\rho_{1}$ and $\rho_{2}$ being parameters). Also, $E\left(u \mid v_{2}, x\right)=E\left(u \mid v_{2}\right)$ may not hold as in a model $E\left(u \mid x, v_{2}\right)=\rho_{1}(x) v_{2}+\rho_{2}(x) v_{2}^{2}$ where the heteroskedasticity factors $\rho_{1}(x)$ and $\rho_{2}(x)$ are functions of $x$.

Terza et al. (2008) advocated the CF approach of controlling $\hat{v}_{2}$, after comparing it with the 'substitution approach (SUB)' of replacing $y_{2}$ with its $x$-predicted version $\hat{y}_{2}$. Kang and Lee (2010), however, recommended SUB after comparing four approaches including CF and SUB. Their recommendation of SUB was made because SUB is the simplest to understand/use with its intuitive appeal; although SUB did worse than others, the differences across the approaches were small. On the other hand, Lee (2012a) made the following recommendation. First, try both SUB and CF. Second, if they differ much, then modify the CF form until SUB and $\mathrm{CF}$ give similar results by trying nonlinear terms of $v_{2}$ or heteroskedasticity factors. Third, conduct inference using CF as its standard error is smaller. Almirall et al. (2010) also used a CF with a heteroskedasticity factor, albeit in a different context (dynamic treatment effects). We will heed to this recommendation in our empirical analysis later.

For an LDV regressor such as $y_{2}$, it is not obvious which form of the $y_{2}$ residual will be the best choice for $\mathrm{CF}$. For a count regressor, there is no 'natural' residual. To motivate our approach to this count case, consider generating a Poisson regressor $y$ with parameter

$$
\exp \left(x^{\prime} \xi+\varepsilon\right)
$$

where $\varepsilon$ is unrelated to $x$ (i.e., $\varepsilon \amalg x$ ) but related to $u$ so that $y$ becomes endogenous for $y_{1}$; for example, $u$ consists of $\varepsilon$ and an additive error. To generate $y$, many exponential random durations with the same parameter $\exp \left(x^{\prime} \xi+\varepsilon\right)$ should be generated first. Then, the number of the exponential durations that can be fit into the unitary time interval is the desired Poisson $y$-after this, the LDV $y_{1}$ can be generated using ( $x$ and) $y$ and $u$. For the endogenous $y$, at least the following two types of residuals can be thought of.

The 'additive residual' for $y$ is

$$
y-E(y \mid x)=y-E\{E(y \mid x, \varepsilon) \mid x\}=y-E\left\{\exp \left(x^{\prime} \xi\right) e^{\varepsilon} \mid x\right\}=y-\exp \left(x^{\prime} \xi\right) \cdot E\left(e^{\varepsilon}\right) \text { due to } \varepsilon \amalg x .
$$

From this, it follows that

$$
\begin{aligned}
E\left[y-\exp \left(x^{\prime} \xi\right) \cdot E\left(e^{\varepsilon}\right) \mid x\right] & =E\left[E\left\{y-\exp \left(x^{\prime} \xi\right) \cdot E\left(e^{\varepsilon}\right) \mid \varepsilon, x\right\} \mid x\right]=E\left[\exp \left(x^{\prime} \xi\right) e^{\varepsilon}-\exp \left(x^{\prime} \xi\right) E\left(e^{\varepsilon}\right) \mid x\right] \\
& =E\left[\exp \left(x^{\prime} \xi\right) \cdot\left\{e^{\varepsilon}-E\left(e^{\varepsilon}\right)\right\} \mid x\right]=0 .
\end{aligned}
$$

Hence, using $y-E(y \mid x)$ amounts to using $\exp \left(x^{\prime} \xi\right) \cdot\left\{e^{\varepsilon}-E\left(e^{\varepsilon}\right)\right\}$ as a CF in the $y_{1}$ SF.

A better choice than the additive residual might be the multiplicative residual $\{y / E(y \mid x)\}-1$, which leads to

$$
E\left\{\frac{y}{E(y \mid x)}-1 \mid x\right\}=E\left[E\left\{\frac{y}{E(y \mid x)}-1 \mid \varepsilon, x\right\} \mid x\right]=E\left(\frac{\exp \left(x^{\prime} \xi\right) e^{\varepsilon}}{\exp \left(x^{\prime} \xi\right) E\left(e^{\varepsilon}\right)}-1 \mid x\right)=0 .
$$

Hence, using $\left\{y_{2} / E(y \mid x)\right\}-1$ is analogous to using $\left\{e^{\varepsilon} / E\left(e^{\varepsilon}\right)\right\}-1$ as a CF in the $y_{1}$ SF. The main difference between the two residuals is that the additive residual carries the heteroskedasticity factor $\exp \left(x^{\prime} \xi\right)$, whereas the multiplicative residual does not.

For $y_{2}=q y_{2}^{*}$, the two types of residual become more complicated. Recalling (2.2), they are not $y_{2}-\exp \left(x^{\prime} \beta\right)$ and $y_{2} \exp \left(-x^{\prime} \beta\right)-1$, but

$$
y_{2}-\frac{\exp \left(x^{\prime} \alpha\right)}{1+\exp \left(x^{\prime} \alpha\right)} \exp \left(x^{\prime} \beta\right) \text { and } y_{2}\left\{\frac{\exp \left(x^{\prime} \alpha\right)}{1+\exp \left(x^{\prime} \alpha\right)} \exp \left(x^{\prime} \beta\right)\right\}^{-1}-1 \text {. }
$$

$\mathrm{CF}$ with these will be examined in detail later. For our empirical analysis, we will try both residuals because which is better will be determined ultimately by how much endogeneity can be picked up by each type of residual; the more the better. 
Because SCL in the second stage needs only the symmetry of $u \mid x$ around zero, the only parametric assumption for our two-stage procedure is the logit in the first stage. Because there is no practical semiparametric estimator for binary responses, assuming logit does not seem so restrictive. If we desire to avoid even the logit assumption, then we may simply assume

$$
E\left(y_{2} \mid x\right)=\exp \left(x^{\prime} \beta\right) .
$$

This will be also applied to our data later, and as it turns outs, its performance is inferior to our two-stage procedure allowing for 'zero inflation'.

\subsection{Second stage with Symmetrically Censored LSE (SCL)}

In our two-stage procedure, the second stage is SCL with a CF used as an extra regressor to remove the $y_{2}$ endogeneity. Here, we explain SCL first, pretending that $y_{2}$ is exogenous for a while. We obtain $y_{1 i}=\max \left(0, w_{i}^{\prime} \gamma+u_{i}\right)$, defining

$$
w \equiv\left(y_{2}, x_{1}^{\prime}\right)^{\prime} \text { and } \gamma \equiv\left(\gamma_{y}, \gamma_{x}^{\prime}\right)^{\prime} .
$$

Observe $0 \leq w^{\prime} \gamma+u \Leftrightarrow-w^{\prime} \gamma \leq u$. If $0<w^{\prime} \gamma$, then the censoring of $y_{1}$ at zero replaces the lower tail of $u$ with a 'mass' $-w^{\prime} \gamma$. The idea of SCL is to artificially replace the upper tail with $w^{\prime} \gamma$ to restore the symmetry of $u$. This leads to the moment condition

$$
E\left\{1\left[w^{\prime} \gamma>0\right] \cdot\left(1\left[|u|<w^{\prime} \gamma\right] u+w^{\prime} \gamma 1\left[|u| \geq w^{\prime} \gamma\right]\right) \cdot w\right\}=0 .
$$

A minimand with this moment condition as its asymptotic first-order condition is

$$
\frac{1}{N} \sum_{i}\left[\left\{y_{1 i}-\max \left(0.5 y_{1 i}, w_{i}^{\prime} \gamma\right)\right\}^{2}+1\left[y_{1 i}>2 w_{i}^{\prime} \gamma\right] \cdot\left\{\left(0.5 y_{1 i}\right)^{2}-\left(\max \left(0, w_{i}^{\prime} \gamma\right)\right)^{2}\right\}\right]
$$

and SCL is obtained by minimizing this for $\gamma$.

If $w_{i}^{\prime} \gamma \simeq \infty \forall i$ (i.e., no censoring problem of $u_{i}$ by $-w_{i}^{\prime} \gamma$ ), then the minimand becomes the LSE minimand $N^{-1} \sum_{i}\left(y_{1 i}-w_{i}^{\prime} \gamma\right)^{2}$. The SCL second-order (Hessian) matrix is

$$
H \equiv E\left(1\left[|u|<w^{\prime} \gamma\right] w w^{\prime}\right)
$$

which becomes $E\left(w w^{\prime}\right)$, the Hessian matrix of LSE, when $|u|<w^{\prime} \gamma$ (this is implied by $w^{\prime} \gamma \simeq \infty$ ). If the censoring proportion becomes small, then SCL becomes close to LSE, and in this sense, SCL is a natural estimator for censored models with a small censoring proportion; such an application has been made, for example, in Levitt (1996). The main advantage of SCL over parametric estimators for censored models is that SCL does not specify the distribution of $u l w$ and allows an unknown form of heteroskedasticity because the aforementioned moment condition does not require $u \amalg w$.

Powell (1986) suggested an iterative scheme to obtain $\hat{\gamma}$. Start with an initial estimate $\hat{\gamma}_{0}$, say LSE, and then iterate the following until convergence:

$$
\hat{\gamma}=\left(\sum_{i} 1\left[w_{i}^{\prime} \hat{\gamma}_{0}>0\right] \cdot w_{i} w_{i}^{\prime}\right)^{-1} \sum_{i}\left\{1\left[w_{i}^{\prime} \hat{\gamma}_{0}>0\right] \min \left(y_{1 i}, 2 w_{i}^{\prime} \hat{\gamma}_{0}\right) \cdot w_{i}\right\} .
$$

This does not guarantee global convergence. Also, the matrix to be inverted may not be invertible. If this problem occurs, then removing $1\left[w_{i}^{\prime} \hat{\gamma}_{0}>0\right]$ in the inverted matrix may help. From our experience, however, this algorithm works well. Santos-Silva (2001) suggested an improved algorithm for SCL. Honoré (1992) extended SCL to 'fixed-effect' panel censored models, and its application can be seen, for example, in Kang and Lee (2003). In Google Scholar, Powell's (1984) censored least absolute deviation estimator has been 
cited 747 times as of this writing, whereas its LSE-version SCL was cited only 322 times. This seems due to the popularity gained by quantile regression relative to the conventional mean regression since 1980s.

Going back to the case with endogenous $y_{2}$, let $v_{2}$ be either the additive or multiplicative residual from the $y_{2}$ $\mathrm{RF}$. Then, the second stage in our two-stage procedure is SCL with $w$ augmented by the CF $\hat{v}_{2}$ (along with $\hat{v}_{2}^{2}$ or $\hat{v}_{2}$ times a heteroskedasticity factor). With the endogeneity of $y_{2}$ removed by the CF, SCL can be implement as earlier. The only modification needed is the asymptotic variance of SCL because the first-stage estimation errors $\hat{\alpha}-\alpha$ and $\hat{\beta}-\beta$ affect the SCL asymptotic variance through $\hat{v}_{2}$, which will be examined in detail shortly.

Our two-stage procedure works well computationally because all estimators involved (logit, QPOI and SCL) converge well. This computational advantage should not be downplayed as it matters greatly in practice.

\subsection{Asymptotic distribution}

When $w$ is exogenous for $y_{1}$, the first-order and second-order derivatives of the SCL minimand give the following asymptotic linear expansion of SCL:

$$
\begin{aligned}
\sqrt{N}(\hat{\gamma}-\gamma) & =\frac{1}{\sqrt{N}} \sum_{i} H^{-1} \cdot 1\left[w_{i}^{\prime} \gamma>0\right]\left(1\left[\left|u_{i}\right|<w_{i}^{\prime} \gamma\right] u_{i}+w_{i}^{\prime} \gamma 1\left[\left|u_{i}\right| \geq w_{i}^{\prime} \gamma\right]\right) w_{i}+o_{p}(1) \\
& =\frac{1}{\sqrt{N}} \sum_{i} H^{-1} \zeta_{i}+o_{p}(1) \text { where } \zeta_{i} \equiv 1\left[w_{i}^{\prime} \gamma>0\right]\left(1\left[\left|u_{i}\right|<w_{i}^{\prime} \gamma\right] u_{i}+w_{i}^{\prime} \gamma 1\left[\left|u_{i}\right| \geq w_{i}^{\prime} \gamma\right]\right) w_{i} .
\end{aligned}
$$

From this, it follows that with ' $w$ ' denoting convergence in law,

$$
\sqrt{N}(\hat{\gamma}-\gamma) w N\left\{0, H^{-1} E\left(\zeta \zeta^{\prime}\right) H^{-1}\right\} \quad \text { where } E\left(\zeta \zeta^{\prime}\right)=E\left\{1\left[w^{\prime} \gamma>0\right] \min \left(u^{2},\left(w^{\prime} \gamma\right)^{2}\right) \cdot w w^{\prime}\right\} .
$$

As already mentioned, the first-stage estimation errors $\hat{\alpha}-\alpha$ and $\hat{\beta}-\beta$ affect the SCL asymptotic variance through $\hat{v}_{2}$, which is discussed now.

Redefine $w$ and $\gamma$ as

$$
w=\left(y_{2}, x_{1}^{\prime}, \hat{v}_{2}, \hat{v}_{2}^{2}, \hat{v}_{2} z\right)^{\prime} \quad \text { and } \quad \gamma=\left(\gamma_{y}, \gamma_{x}^{\prime}, \gamma_{1}, \gamma_{2}, \gamma_{z}^{\prime}\right)^{\prime}
$$

where $z$ represents the elements of $x$ that interact with $v_{2}, \hat{v}_{2}=\hat{v}_{2}(\hat{\alpha}, \hat{\beta})$ that depends on $\hat{\alpha}$, and $\hat{\beta}$ is either the additive or multiplicative residual, and $\left(\gamma_{1}, \gamma_{2}, \gamma_{z}^{\prime}\right)^{\prime}$ is the coefficient vector for $\left(\hat{v}_{2}, \hat{v}_{2}^{2}, \hat{v}_{2} z\right)$. Here, $\hat{v}_{2}^{2}$ and $\hat{v}_{2} z$ are used in addition to $\hat{v}_{2}$, as all three $\left(\hat{v}_{2}, \hat{v}_{2}^{2}, \hat{v}_{2} z\right)$ will appear in our empirical analysis later.

The presence of the first-stage estimators $\hat{\alpha}$ and $\hat{\beta}$ matters for the 'gradient vector' $\zeta$ in the earlier linear expansion of SCL but not for the second-order matrix $H$. Hence, rewrite the asymptotic linear expansion as

$$
\begin{aligned}
\sqrt{N}(\hat{\gamma}-\gamma) & =\frac{1}{\sqrt{N}} \sum_{i} H^{-1} \zeta_{i}(\hat{\alpha}, \hat{\beta})+o_{p}(1) \\
& =\frac{1}{\sqrt{N}} \sum_{i} H^{-1}\left\{\zeta_{i}(\alpha, \beta)+E\left(\zeta_{\alpha^{\prime}}\right) \eta_{\alpha i}+E\left(\zeta_{\beta^{\prime}}\right) \eta_{\beta i}\right\}+o_{p}(1)
\end{aligned}
$$

where $\zeta_{\alpha^{\prime}}$ and $\zeta_{\beta^{\prime}}$ denote the derivatives of $\zeta(\alpha, \beta)$ for $\alpha$ and $\beta$, respectively, and $\eta_{\alpha i}$ and $\eta_{\beta i}$ are 'influence functions' for $\hat{\alpha}$ and $\hat{\beta}$ :

$$
\begin{aligned}
& \eta_{\alpha i}=\left\{E\left(s s^{\prime}\right)\right\}^{-1} s_{i} \quad \text { for logit score function } s_{i}=\left\{y_{2 i}-\frac{\exp \left(x_{i}^{\prime} \alpha\right)}{1+\exp \left(x_{i}^{\prime} \alpha\right)}\right\} x_{i}, \\
& \eta_{\beta i}=\left[E\left\{q x x^{\prime} \exp \left(x^{\prime} \beta\right)\right\}\right]^{-1} q_{i} x_{i}\left\{y_{2 i}-\exp \left(x_{i}^{\prime} \beta\right)\right\} .
\end{aligned}
$$

For instance, when $\mathrm{z}$ is a scalar, the dimension of $\gamma$ is $\left(k_{1}+4\right) \times 1$ and the dimensions of $\alpha$ and $\beta$ are both $k \times 1$. Then $\zeta_{\alpha^{\prime}}$ and $\zeta_{\beta^{\prime}}$ are $\left(k_{1}+4\right) \times k$ matrices, which can be obtained by numerical differentiation. See, for example, Lee (2010) for more details on this way of accounting for the first-stage estimation errors. 
From the asymptotic linear expansion taking into account $\hat{\alpha}-\alpha$ and $\hat{\beta}-\beta$, we obtain

$$
\sqrt{N}(\hat{\gamma}-\gamma) \leftrightarrow N\left\{0, H^{-1} E\left(\lambda \lambda^{\prime}\right) H^{-1}\right\} \quad \text { where } \lambda_{i} \equiv \zeta_{i}(\alpha, \beta)+E\left(\zeta_{\alpha^{\prime}}\right) \eta_{\alpha i}+E\left(\zeta_{\beta^{\prime}}\right) \eta_{\beta i} .
$$

$E\left(\lambda \lambda^{\prime}\right)$ can be estimated consistently by replacing $(\alpha, \beta, \gamma)$ with $(\hat{\alpha}, \hat{\beta}, \hat{\gamma})$ and the expected values in $\lambda$ with the corresponding sample means. If $E\left(y_{2} \mid x\right)=\exp \left(x^{\prime} \beta\right)$ is adopted, then the only required change is redefining $v_{2}$ without the logit probability and then removing $E\left(\zeta_{\alpha^{\prime}}\right) \eta_{\alpha i}$ in $\lambda$. The endogeneity of $y_{2}$ can be tested using $\left(\hat{\gamma}_{1}, \hat{\gamma}_{2}, \hat{\gamma}_{z}^{\prime}\right)$, as these should be all zero under the null of $y_{2}$ exogeneity.

It is interesting to note that although we toiled to account for the first-stage estimation errors $\hat{\alpha}-\alpha$ and $\hat{\beta}-\beta$, they can be ignored for SCL under the null of $y_{2}$ exogeneity because the effect of the estimation errors $\hat{\alpha}-\alpha$ and $\hat{\beta}-\beta$ operates through $\left(\hat{\gamma}_{1}, \hat{\gamma}_{2}, \hat{\gamma}_{z}^{\prime}\right)^{\prime}$.

\subsection{Details on control function}

In practice, it may be enough for a CF based on (2.3) to carry a significant estimate so that the results under $y_{2}$ exogeneity assumption differ much from those allowing $y_{2}$ endogeneity. But it would be more desirable to know what the CF really looks like and to justify it properly. Here, we take a detailed look at the CF's under more assumptions.

For a parameter vector $\tilde{\beta}$ and an error $\varepsilon$ possibly related to $u$, make an extra assumption

$$
E\left(y_{2}^{*} \mid x, \varepsilon, q=1\right)=\exp \left(x^{\prime} \tilde{\beta}+\varepsilon\right) \text { and } \varepsilon \amalg(x, q) \Leftrightarrow \varepsilon \amalg q \mid x \text { and } \varepsilon \amalg x .
$$

This implies our earlier model assumptions in (2.1):

$$
\begin{aligned}
E(q=1 \mid x, \varepsilon) & =P(q=1 \mid x)\left(=\frac{\exp \left(x^{\prime} \alpha\right)}{1+\exp \left(x^{\prime} \alpha\right)}\right) \text { due to } \varepsilon \amalg q \mid x ; \\
E\left(y_{2}^{*} \mid x, q=1\right) & =\int E\left(y_{2}^{*} \mid x, \varepsilon, q=1\right) f(\varepsilon \mid x, q=1) d \varepsilon=\exp \left(x^{\prime} \tilde{\beta}\right) \int e^{\varepsilon} f(\varepsilon) d \varepsilon \text { due to } \varepsilon \amalg(x, q) \\
& =\exp \left(x^{\prime} \tilde{\beta}\right) \cdot E\left(e^{\varepsilon}\right)=\exp \left\{x^{\prime} \tilde{\beta}+\ln E\left(e^{\varepsilon}\right)\right\}=\exp \left(x^{\prime} \beta\right)
\end{aligned}
$$

where $f(\varepsilon \mid x, q=1)$ denotes the density of $\varepsilon \mid(x, q=1)$ and $\beta$ differs from $\tilde{\beta}$ only in that the intercept in $\beta$ equals the intercept in $\tilde{\beta}$ plus $\ln E\left(e^{\varepsilon}\right)$.

Noting

$$
E\left(y_{2} \mid x, \varepsilon\right)=E\left(q y_{2}^{*} \mid x, \varepsilon\right)=P(q=1 \mid x, \varepsilon) E\left(y_{2}^{*} \mid x, \varepsilon, q=1\right)=\frac{\exp \left(x^{\prime} \alpha\right)}{1+\exp \left(x^{\prime} \alpha\right)} \exp \left(x^{\prime} \tilde{\beta}\right) e^{\varepsilon},
$$

the reason for the extra assumption (2.4) can be seen in $E$ \{additive residual of (2.3)|x\}:

$$
\begin{aligned}
E\left\{y_{2}-\frac{\exp \left(x^{\prime} \alpha\right)}{1+\exp \left(x^{\prime} \alpha\right)} \exp \left(x^{\prime} \beta\right) \mid x\right\} & =E\left[E\left\{y_{2}-\frac{\exp \left(x^{\prime} \alpha\right)}{1+\exp \left(x^{\prime} \alpha\right)} \exp \left(x^{\prime} \tilde{\beta}\right) E\left(e^{\varepsilon}\right) \mid x, \varepsilon\right\} \mid x\right] \\
& =E\left[\frac{\exp \left(x^{\prime} \alpha\right)}{1+\exp \left(x^{\prime} \alpha\right)} \exp \left(x^{\prime} \tilde{\beta}\right) \cdot\left\{e^{\varepsilon}-E\left(e^{\varepsilon}\right)\right\} \mid x\right]=0 .
\end{aligned}
$$

That is, using the additive residual $\mathrm{CF}$ amounts to using

$$
\frac{\exp \left(x^{\prime} \alpha\right)}{1+\exp \left(x^{\prime} \alpha\right)} \exp \left(x^{\prime} \tilde{\beta}\right) \cdot\left\{e^{\varepsilon}-E\left(e^{\varepsilon}\right)\right\} \text {. }
$$

Doing analogously, using the multiplicative residual CF amounts to using $\left\{e^{\varepsilon} / E\left(e^{\varepsilon}\right)\right\}-1$.

Because $y_{2}$ should be exogenous once $\varepsilon$ is controlled via a CF, the relation of $\varepsilon$ to $u$ should be the only source for the $y_{2}$ endogeneity. If $\varepsilon$ were the only error in $y_{2}^{*}$ given $x$, then ' $\varepsilon \amalg(x, q)$ ' in (2.4) would imply the 'outcome 
equation' $y_{2}^{*}$ and the selection equation $q$ being independent given $x$ - 'selection on observables'. But there is an error other than $\varepsilon$ that makes $y_{2}^{*}$ 'random' given $(x, \varepsilon, q=1)$, as can be seen in the expression $E\left(y_{2}^{*} \mid x, \varepsilon, q=1\right)$. Hence, the selection-on-observable between $y_{2}^{*}$ and $q$ in $y_{2}=q y_{2}^{*}$ given $x$ is not implied by $\varepsilon \amalg(x, q)$. It should be noted, however, that (2.4) requires the endogeneity of $y_{2}$ to come from $y_{2}^{*}$ not from $q$. To see this, imagine $q$ and $\varepsilon$ both related to $u$ given $x$, which implies a relation between $q$ and $\varepsilon$ (through $u$ ) to violate $\varepsilon \amalg q \mid x$; thus, $q \coprod u \mid x$ should hold (no endogeneity of $y_{2}$ due to $q$ ), even when $\varepsilon$ is related to $u$ given $x$ (endogeneity of $y_{2}$ due to $y_{2}^{*}$ ).

\subsection{Two-part approach in the literature}

It is helpful to compare our two-stage procedure with the two-part approach in the literature. The two-part approach assumed

$$
\begin{aligned}
& \text { first part : } 1\left[y_{1}^{*}>0\right]=1\left[\gamma_{y} y_{2}+x^{\prime}{ }_{1} \gamma_{x}+u>0\right] \text { and } y_{2}=x^{\prime} \delta+v \\
& \text { second part }: y_{1}=\xi_{y} y_{2}+x_{1}^{\prime} \xi_{x}+e \text { given } y_{1}>0
\end{aligned}
$$

where $\delta$ and $\xi$ are parameters and $v$ and $e$ are error terms.

For the first part, substitute $y_{2}=x^{\prime} \delta+v$ to obtain

$$
\begin{aligned}
& 1\left[y_{1}^{*}>0\right]=1\left[\gamma_{y}\left(x^{\prime} \delta+v\right)+x^{\prime} S \gamma_{x}+u>0\right]=1\left[x^{\prime} \psi+\gamma_{y} v+u>0\right] \\
& \text { where } \psi \equiv \gamma_{y} \delta+S \gamma_{x} \text { and } S \text { consists of } 0 \text { s and } 1 \text { s such that } x_{1}^{\prime}=x^{\prime} S
\end{aligned}
$$

$\psi$ is the RF parameters for $1\left[y_{1}^{*}>0\right]$, whereas $\left(\gamma_{y}, \gamma_{x}\right)$ is the SF parameters. For the endogeneity of $y_{2}$ in the first part, a CF approach combined with minimum distance estimator (MDE) was used by the two-part approach: the LSE residual $\hat{v}$ for the $y_{2}$ equation is used along with $x$ to obtain $\left(\hat{\psi}, \hat{\gamma}_{y}\right)$ for $(2.5)$, and then $\left(\delta, \gamma_{x}\right)$ is estimated by MDE using $\hat{\psi} \simeq \hat{\gamma}_{y} \delta+S \gamma_{x}$-simply imagine the LSE of $\hat{\psi}$ on $\left(\hat{\gamma}_{y}, S\right)$ to estimate $\left(\delta, \gamma_{x}\right)$.

Some remarks on the two-part approach are in order. First, $\left(\gamma_{y}, \gamma_{x}\right)$ can be estimated in the $1\left[y_{1}^{*}>0\right]$ SF with $\hat{v}$ controlled; no MDE is needed. Second, the linear model for $y_{2}$ is not plausible as $y_{2}$ has many zeros. Third, the second part of the two-part approach has been 'sold' (relative to sample selection models) for a better prediction of $y_{1}$; hence, the second part is not suitable to allow for an endogenous regressor such as $y_{2}$.

\section{EMPIRICAL ANALYSIS}

The national health insurance program in Korea covers the entire population as a compulsory social insurance. It is funded by the insured and the government subsidy. If desired, each individual can obtain supplementary private health insurances that reduce the copayment for the national health insurance services. Also, a national long-term care insurance was introduced in 2008 that has provisions supporting unpaid family caregivers in two ways: family caregivers in remote areas where long-term-care licensees cannot reach get paid fully for their family care and those in accessible areas get paid up to two hours of service per day. As in other developed countries, fiscal sustainability is a big concern for the Korean national health insurance because of the fastaging population and ever-increasing demand for health care services. Hence, there exists a strong motive to reduce the formal health care cost.

Our data was drawn from the elderly of age 65 or above in 'the Korean Longitudinal Study of Ageing' for the year 2008. The information on the variables can be found in Table I. In Table I, 'formal' is the annual medical and long-term care expenditure in about $\$ 1000$ - the other amounts in the table are all annual amounts in the same unit. The number of caregivers is the informal family care variable, $85 \%$ of which are zeros. Table I also shows yearly informal care hours ('care hours') of which $85 \%$ are zeros again, but this variable will not be used for $y_{2}$-the estimation results with care hours as $y_{2}$ is mostly insignificant with no endogeneity of $y_{2}$ picked up by the CFs. 
Table I. Descriptive statistics

\begin{tabular}{|c|c|c|c|c|c|}
\hline Variable & Mean (SD) & Min,Max & Variable & Mean (SD) & Min,Max \\
\hline Formal $(\$ 1000)$ & 1.179 (2.34) & $0,48.4$ & Age & $74.6(6.12)$ & 65,107 \\
\hline \# caregivers & $0.215(0.58)$ & 0,4 & Male & $0.425(0.494)$ & 0,1 \\
\hline Care hours & 157 (619) & 0,8760 & Married & $0.636(0.481)$ & 0,1 \\
\hline Fi. asset $(\$ 1000)$ & 4.88 (21.6) & 0,500 & Seoul & $0.137(0.343)$ & 0,1 \\
\hline Real est. (\$1000) & $152(222)$ & 0,2948 & Work & $0.213(0.409)$ & 0,1 \\
\hline Own house & $0.409(0.49)$ & 0,1 & kid-par (\$1000) & $13.5(28.2)$ & 0,866 \\
\hline Fam.inc. (\$1000) & $16.3(21.0)$ & 0,700 & nkids & $3.99(1.61)$ & 0,10 \\
\hline Pension (\$1000) & $1.42(4.44)$ & $0,94.9$ & nfem.kids & $1.92(1.40)$ & 0,8 \\
\hline Hi.bl. pressure & $0.091(0.288)$ & 0,1 & nkids-co & $0.412(0.56)$ & 0,3 \\
\hline Diabetes & $0.048(0.215)$ & 0,1 & nfem.kids-co & $0.092(0.30)$ & 0,3 \\
\hline Cancer/tumor & $0.013(0.114)$ & 0,1 & nkids-act & $2.61(1.41)$ & 0,8 \\
\hline Chronic pulmo & $0.016(0.127)$ & 0,1 & nfem.kids-act & $0.765(0.97)$ & 0,7 \\
\hline Chronic liver & $0.005(0.073)$ & 0,1 & nkids-30 & $0.597(0.99)$ & 0,6 \\
\hline Cardio disease & $0.035(0.183)$ & 0,1 & nkids-60 & $0.838(1.18)$ & 0,6 \\
\hline Cerebral bl.vessel & $0.038(0.191)$ & 0,1 & nkids-120 & $0.768(1.22)$ & 0,9 \\
\hline Mental disease & $0.016(0.125)$ & 0,1 & \# generations & $1.48(1.06)$ & 0,4 \\
\hline Arthritis/rheuma. & $0.195(0.396)$ & 0,1 & PRO-fem & $0.452(0.267)$ & 0,1 \\
\hline
\end{tabular}

The variable 'fi. asset' is the financial asset amount, 'real est.' is the real asset amount, 'own house' is the dummy for owning a house, and 'fam.inc.' is the household income. Pension is the pension and other welfare receipt amount. 'hi.bl. pressure' is the dummy for high blood pressure, 'cancer/tumor' for cancer or malign tumors, 'chronic pulmo.' for chronic pulmonary diseases, 'chronic liver' for chronic liver diseases, 'cerebral bl.vessel' for cerebral blood vessel diseases, and 'arthritis/rheuma.' for arthritis or rheumatism. 'Seoul' is the dummy for living in Seoul, and 'work' for working. 'kid-par' is the transfer amount from children to the parents. 'nkids' is the number of children, 'nfem.kids' for the daughters, 'nkids-co' for the cohabiting children with the respondent, 'nkids-act' for the children economically active, and 'nfem.kids-act' for the daughters economically active. 'nkids-30' is the number of noncohabiting children living in 1-30 min by public transportation; nkids-60 and nkids-120 are analogously defined for 31-60 and 61-120 min, respectively. '\# generations' is the number of generations living together. 'pro-fem' is the proportion of female children defined as 'nfem.kids/nkids' if nkids is positive and 0 otherwise.

We apply $\ln (\cdot+1)$ transformation to all amount variables, which has a number of advantages. First, logarithmic transformation removes extreme values and tends to equalize the scales of the variables. Second, logarithmic transformation tends to remove the error term heteroskedasticity and make the error term distribution symmetric. Third, although adding 1 makes hardly any difference to the amount variables, it preserves the censoring aspect (or more precisely, the corner solution aspect) because zero remains zero after the transformation and positive values remain positive.

Santos-Silva and Tenreyro (2006) noted that logarithmic transformation may cause inconsistency, but their argument is based on $E(y \mid x)$ being linear not $E(\ln y \mid x)$; broadly viewed, this is the 'transformation of variables' issue for the best transformation $\tau(y)$ such that $E(\tau(y) \mid x)$ is linear. Santos-Silva and Tenreyro suggested QPOI under an exponential regression, say $E(y \mid x)=\exp \left(x^{\prime} \gamma\right)$. This is fine as far as approximating $E(y \mid x)$ with $\exp \left(x^{\prime} \gamma\right)$ goes, knowing $y \geq 0$, but it falls short if one tries to generate a continuously distributed $y>0$ : it is hard, if not impossible, to 'smoothly generate' $y$ subject to $E(y \mid x)=\exp \left(x^{\prime} \gamma\right)$ and $P(y=0 \mid x)>0$, unless an abrupt censoring/selection mechanism is allowed.

Other than the variables in Table I, self-reported health status is also available in five categories. But when health status was used for estimation, its coefficient was significantly positive, implying that health status is likely to be affected by formal/informal care, and thus, it cannot be used as a regressor. Although the children-related variables can be used as instruments (IV) for $y_{2}$, there is no good IV for health status. Hence, health status is dropped from the regressor list. By omitting health status, the $y_{2}$ endogeneity becomes more likely. 
Although we drop the health status, we keep the nine illness dummies and use their interactions with $y_{2}$ because the degree of the substitutability of $y_{2}$ for $y_{1}$ is likely to depend on those illnesses. But this entails a restriction that those illness dummies are exogenous whereas the health status is not. One justification for the restriction is that self-reported health status should be more easily influenced by formal/informal care, whereas those illnesses tend to be chronic and thus not as easily influenced or cured.

To appreciate the consequences of omitting health status, consider a linear model for positive health status $h$ and a linear $y_{1}$ SF with $h$ explicit:

$$
h=\theta_{1} y_{1}+\theta_{2} y_{2}+x^{\prime} \theta_{x}+\kappa\left(\theta_{1}, \theta_{2}>0\right) \text { and } \quad y_{1}=\gamma_{h} h+\gamma_{y} y_{2}+x_{1}^{\prime} \gamma_{x}+u\left(\gamma_{h}<0\right)
$$

where $\theta \mathrm{s}$ are parameters and $\kappa$ is an error term; ' $\theta_{1}, \theta_{2}>0$ ' means improved health with health care, and ' $\gamma_{h}<0$ ' means the lesser formal care for the healthier. Substitute the $h$ equation into the $y_{1}$ equation and then solve for $y_{1}$ :

$$
\begin{aligned}
y_{1} & =\gamma_{h}\left(\theta_{1} y_{1}+\theta_{2} y_{2}+x^{\prime} \theta_{x}+\kappa\right)+\gamma_{y} y_{2}+x_{1}^{\prime} \gamma_{x}+u \\
& =\gamma_{h} \theta_{1} y_{1}+\left(\gamma_{h} \theta_{2}+\gamma_{y}\right) y_{2}+x^{\prime} \gamma_{h} \theta_{x}+x_{1}^{\prime} \gamma_{x}+\left(\gamma_{h} \kappa+u\right) \\
\Leftrightarrow y_{1} & =\frac{1}{1-\gamma_{h} \theta_{1}}\left\{\left(\gamma_{h} \theta_{2}+\gamma_{y}\right) y_{2}+x^{\prime} \gamma_{h} \theta_{x}+x_{1}^{\prime} \gamma_{x}+\left(\gamma_{h} \kappa+u\right)\right\} .
\end{aligned}
$$

The interest is on the following effects of $y_{2}$ on $y_{1}: \gamma_{y}$ (direct effect with $h$ controlled) versus $\gamma_{y}^{*} \equiv$ $\frac{\gamma_{h} \theta_{2}+\gamma_{y}}{1-\gamma_{h} \theta_{1}}$ (total effect with $h$ substituted out);only $\gamma_{y}^{*}$ is identified by dropping $h$, although the desired effect might be $\gamma_{y}$-but one may 'declare' that $\gamma_{y}^{*}$ is the desired effect. Because $1-\gamma_{h} \theta_{1}>1$, the sign of $\gamma_{y}^{*}$ depends on the sign of $\gamma_{h} \theta_{2}+\gamma_{y}$ that consists of the direct effect $\gamma_{y}$ of $y_{2}$ on $y_{1}$ and the 'indirect effect' $\gamma_{h} \theta_{2}<0$ through the improved health; see Huang and Lee (2010) and Lee and Huang (2012) for this type of decomposition of a total effect into the direct and indirect effects. Because $\gamma_{h} \theta_{2}<0, \gamma_{y}<0$ implies $\gamma_{h} \theta_{2}+\gamma_{y}<0 ; \gamma_{y}>0$, however, makes the sign of $\gamma_{h} \theta_{2}+\gamma_{y}$ ambiguous. That is, $\gamma_{y}^{*}<0$ does not necessarily imply $\gamma_{y}<0$, but $\gamma_{y}^{*}>0$ implies $\gamma_{y}>0$.

Table II 'logit and quasi Poisson for $y_{2}$ ' presents the estimates for the first stage. Because most disease variables are highly significant but of no direct interest, we omit their results in Table II and in the remaining tables to simplify presentation; also omitted are the intercept estimates. In Table II, age $2 / 100$ ('age2') is used as well. The main variables of interest are the children-related variables as they are the IVs for $y_{2}$ and thus should be significant in explaining $y_{2}$. \# generations and possibly nkids and nfem.kids-co are significant for logit, whereas nfem.kidsact, nkids-30, nkids-60, and pro-fem are significant for QPOI. Nevertheless, the following limitations of the IVs should be noted. First, having many kids (i.e., a high nkids) may result in a poor unobserved health $\kappa$ that is part of the $y_{1}$ equation error as can be seen in (3.1). Second, nfem.kids-co and nfem.kids-act may be affected by $\kappa$ because children may withdraw from the labor market to take care of their parents; in the analogous way, nfem. kids-co may be related to $\kappa$. Third, nkids-30 and nkids-60 may be also related to $\kappa$ because children may move closer to their parents to take care of them.

Table III presents the main estimation results where 'tv' stands for $t$-value, CFEa is the estimates with the additive error for CF, CFEm is the estimates with the multiplicative error for CF, and 'tv2' is the correct $t$-value taking into account the first-stage estimation errors, whereas 'tv1' is the $t$-value ignoring the first-stage estimation errors (correct only under the null of $y_{2}$ exogeneity). For the sake of comparison, we show the SCL results ignoring the $y_{2}$ endogeneity in the first column, although we will not interpret the results. As noted already, some variables including 1 were used in estimation, but their results are not shown in Table III: all illness variables and the interaction between $y_{2}$ and the following illnesses: 'cancer/tumor', 'chronic pulmo.', 'chronic liver', 'cardio disease' and 'cerebral bl.vessel'.

Comparing CFEa and CFEm in Table III, CFEm does not pick up the $y_{2}$ endogeneity as the CF's $\left(\hat{v}_{2}, \hat{v}_{2}^{2}, \hat{v}_{2} z\right)$ are all insignificant where $z=(\text { age, male, married })^{\prime}$ - the Wald test for $H_{0}: \gamma_{1}=\gamma_{2}=0$ and $\gamma_{z}=0$ is not rejected. As the result, the CFEm column differs little from the SCL column. In contrast, CFEa does pick 


\section{M.-J. LEE AND Y.-S. KIM}

Table II. Logit and quasi Poisson for $y_{2}$

\begin{tabular}{lcr}
\hline Variables & Logit $(t$-value $)$ & QPOI $(t$-value $)$ \\
\hline Financial asset & $-0.034(-1.54)$ & $-0.011(-1.31)$ \\
Real estate & $0.010(0.27)$ & $0.002(0.08)$ \\
Own house & $-0.244(-1.62)$ & $-0.156(-2.69)$ \\
Family income & $0.057(1.06)$ & $0.030(1.41)$ \\
Pension & $0.025(0.90)$ & $-0.012(-1.19)$ \\
Age & $-0.072(-0.48)$ & $-0.016(-0.25)$ \\
Age2 & $0.111(1.18)$ & $0.014(0.34)$ \\
Male & $0.661(4.02)$ & $0.091(1.46)$ \\
Married & $0.122(0.71)$ & $-0.066(-0.82)$ \\
Seoul & $-0.707(-3.67)$ & $0.036(0.51)$ \\
Work & $-0.821(-3.79)$ & $0.052(0.70)$ \\
kid-par & $-0.051(-2.47)$ & $-0.007(-0.96)$ \\
nkids & $0.209(1.76)$ & $-0.014(-0.34)$ \\
nfem.kids & $-0.142(-0.94)$ & $0.100(1.99)$ \\
nkids-co & $0.095(0.60)$ & $0.066(1.03)$ \\
nfem.kids-co & $0.362(1.76)$ & $0.080(1.22)$ \\
nkids-act & $-0.150(-1.46)$ & $-0.023(-0.60)$ \\
nfem.kids-act & $0.011(0.09)$ & $-0.086(-1.88)$ \\
nkids-30 & $0.040(0.60)$ & $0.049(2.23)$ \\
nkids-60 & $0.023(0.41)$ & $0.044(2.17)$ \\
nkids-120 & $-0.032(-0.54)$ & $-0.010(-0.50)$ \\
\# generations & $0.226(2.91)$ & $0.047(1.52)$ \\
pro-fem & $-0.169(-0.36)$ & $-0.429(-2.60)$ \\
net & &
\end{tabular}

QPOI, quasi-Poisson maximum likelihood estimator.

Table III. SCL, CFE-additive and CFE-multiplicative for $y_{1}$

\begin{tabular}{lcrr}
\hline Variables & SCL (tv) & CFEa (tv2, tv1) & CFEm (tv2, tv1) \\
\hline$y_{2} \times$ hi.bl. pressure & $2.31(2.51)$ & $-4.72(-1.76,-1.88)$ & $2.24(1.57,1.71)$ \\
$y_{2} \times$ iabetes & $-0.22(-1.57)$ & $-0.23(-1.72,-1.73)$ & $-0.21(-1.43,-1.45)$ \\
$y_{2} \times$ mental disease & $-0.68(-3.78)$ & $-0.70(-4.06,-4.10)$ & $-0.68(-3.67,-3.80)$ \\
$y_{2} \times$ marthitis/rheuma. & $-0.48(-1.38)$ & $-0.65(-1.83,-1.84)$ & $-0.49(-1.44,-1.44)$ \\
$y_{2} \times$ arte & $0.10(0.66,0.66)$ & $0.14(0.87,0.91)$ \\
$y_{2} \times$ age & $0.13(0.86)$ & $0.06(1.79,1.92)$ & $-0.03(-1.54,-1.69)$ \\
$y_{2} \times$ male & $-0.03(-2.46)$ & $0.51(1.62,1.69)$ & $0.16(0.86,0.87)$ \\
Financial asset & $0.21(1.32)$ & $0.04(3.04,3.05)$ & $0.05(3.38,3.38)$ \\
Real estate & $0.05(3.40)$ & $0.16(4.72,4.75)$ & $0.16(4.58,4.58)$ \\
Own house & $0.16(4.59)$ & $-0.05-(0.43,-0.43)$ & $-0.03(-0.30,-0.30)$ \\
Family income & $-0.03(-0.28)$ & $0.01(0.36,0.37)$ & $0.00(0.02,0.02)$ \\
Pension & $0.00(0.04)$ & $0.07(3.47,3.47)$ & $0.07(3.47,3.48)$ \\
Age & $0.07(3.47)$ & $0.72(3.35,3.52)$ & $0.38(2.23,2.26)$ \\
Age 2 & $0.37(2.26)$ & $-0.50(-3.44,-3.63)$ & $-0.26(-2.34,-2.38)$ \\
Male & $-0.26(-2.40)$ & $-0.17(-1.18,-1.20)$ & $-0.13(-1.08,-1.08)$ \\
Married & $-0.14(-1.19)$ & $0.09(0.83,0.83)$ & $0.09(0.90,0.90)$ \\
Seoul & $0.09(0.89)$ & $-0.02(-0.16,-0.16)$ & $-0.01(-0.07,-0.07)$ \\
Work & $-0.01(-0.06)$ & $-0.22(-1.87,-1.88)$ & $-0.19(-1.63,-1.63)$ \\
kid-par & $-0.18(-1.61)$ & $0.02(1.52,1.53)$ & $0.03(1.81,1.82)$ \\
$\hat{v}_{2}$ & $0.03(1.81)$ & $7.21(2.62,2.80)$ & $0.01(0.01,0.02)$ \\
$\hat{v}_{2}^{2}$ & & $0.01(0.10,0.10)$ & $0.00(0.21,0.28)$ \\
$\hat{v}_{2} \times$ age & & $-0.09(-2.69,-2.88)$ & $0.00(-0.05,-0.06)$ \\
$\hat{v}_{2} \times$ male & & $-0.56(-1.51,-1.52)$ & $0.01(0.32,0.36)$ \\
$\hat{v}_{2} \times$ married & $0.34(1.53,1.48)$ & $0.00(0.12,0.15)$ \\
\hline
\end{tabular}

SCL, symmetrically censored least squares estimator; CFEa, estimates with the additive error for control function; CFEm, estimates with the multiplicative error for control function. 
up the $y_{2}$ endogeneity because at least $\hat{v}_{2}$ and $\hat{v}_{2} \times$ age are significant, which results in appreciable differences between SCL and CFEa in the estimates involving $y_{2}$. The greatest difference between SCL and CFEa can be seen in the slopes for $y_{2}: 2.31$ for SCL (significant) versus -4.72 for CFEa (nearly significant). In the CFEa column, among the terms involving $y_{2}$, only the interaction term with diabetes is significant with a large effect estimate (70\% reduction in $y_{1}$ as $y_{2}$ goes up by 1 ); there are also weak evidences that $y_{2}$ interacts with high blood pressure, mental disease, age, and male. The poor performance of CFEm relative to CFEa is surprising given the intuitive appeal of the multiplicative residual in the exponential model. This might be attributed to $u$ containing a heteroskedasticity factor present in the additive residual but not in the multiplicative residual.

Also, notable in Table III is that tv2 and tv1 differ little, which means that, in practice, it may be safe to ignore the first-stage correction for the asymptotic variance. It should be mentioned that, in an earlier version of this paper, we used $\left(\hat{v}_{2}, \hat{v}_{2}^{2}, \hat{v}_{2}^{3}\right)$ instead of $\left(\hat{v}_{2}, \hat{v}_{2}^{2}, \hat{v}_{z} z\right)$ to have significant slopes for all of $\left(\hat{v}_{2}, \hat{v}_{2}^{2}, \hat{v}_{2}^{3}\right)$ and rather different tv2 and tv1 for CFEm. But once $\hat{v}_{z} z$ gets used, not just $\hat{v}_{2}^{2}$ and $\hat{v}_{2}^{3}$ lost their significance but also the difference between tv2 and tv1 for CFEm all but disappeared. It is well warranted that practitioners pay more attention to modeling the CF form in terms of using terms such as $\hat{v}_{2} z$ as well as higher-order terms of $\hat{v}_{2}$, instead of using only $\hat{v}_{2}$.

It should be noted that the slope $\hat{v}_{2}$ is large with almost the same scale as that of $y_{2}$. Also, the slopes of $\hat{v}_{2} \times$ age and $\hat{v}_{2} \times$ male have similar scales as those of $y_{2} \times$ age and $y_{2} \times$ male. As for the interaction terms between $y_{2}$ and illnesses, certain informal care at home may be more effective in alleviating certain diseases. For instance, an important part of diabetes treatment is diet, and having a good diet for diabetes may be easily carried out at home by informal care. The same may hold for high blood pressure and mental disease, but perhaps there is very little that can be done for arthritis/rheumatism at home.

Table IV presents the estimation results under $E\left(y_{2} \mid x\right)=\exp \left(x^{\prime} \beta\right)$ that does away with logit; that is, QPOI is applied to $y_{2}$. CFEa still picks up the $y_{2}$ endogeneity in view of the $t$-values of the CFs, but the contrast between SCL and CFEa is weaker than that in Table III as can be seen in the reduced differences in the estimates and $t$-values. CFEm still fails to pick the $y_{2}$ endogeneity.

Table IV. SCL, CFEa and CFEm for $y_{1}$ : no logit

\begin{tabular}{lccr}
\hline Variables & SCL (tv) & CFEa (tv2, tv1) & CFEm (tv2, tv1) \\
\hline$y_{2}$ & $2.31(2.51)$ & $-3.78(-1.32,-1.38)$ & $2.03(1.32,1.41)$ \\
$y_{2} \times$ hi.bl. pressure & $-0.22(-1.57)$ & $-0.26(-1.86,-1.88)$ & $-0.21(-1.42,-1.44)$ \\
$y_{2} \times$ diabetes & $-0.68(-3.78)$ & $-0.68(-3.97,-3.97)$ & $-0.68(-3.58,-3.77)$ \\
$y_{2} \times$ mental disease & $-0.48(-1.38)$ & $-0.67(-1.75,-1.77)$ & $-0.50(-1.45,-1.46)$ \\
$y_{2} \times$ arthritis/rheuma. & $0.13(0.86)$ & $0.13(0.82,0.82)$ & $0.15(0.90,0.95)$ \\
$y_{2} \times$ age & $-0.03(-2.46)$ & $0.04(1.29,1.35)$ & $-0.02(-1.31,-1.41)$ \\
$y_{2} \times$ male & $0.21(1.32)$ & $0.41(1.26,1.30)$ & $0.16(0.75,0.79)$ \\
Financial asset & $0.05(3.40)$ & $0.04(2.97,2.99)$ & $0.05(3.38,3.38)$ \\
Real estate & $0.16(4.59)$ & $0.16(4.85,4.85)$ & $0.16(4.61,4.60)$ \\
Own house & $-0.03(-0.28)$ & $-0.06(-0.58,-0.59)$ & $-0.03(-0.31,-0.31)$ \\
Family income & $0.00(0.04)$ & $0.02(0.54,0.55)$ & $0.00(0.04,0.05)$ \\
Pension & $0.07(3.47)$ & $0.07(3.51,3.51)$ & $0.07(3.45,3.46)$ \\
Age & $0.37(2.26)$ & $0.62(2.95,3.08)$ & $0.38(2.21,2.25)$ \\
Age 2 & $-0.26(-2.40)$ & $-0.43(-3.04,-3.17)$ & $-0.27(-2.32,-2.37)$ \\
Male & $-0.14(-1.19)$ & $-0.14(-1.05,-1.06)$ & $-0.13(-1.05,-1.05)$ \\
Married & $0.09(0.89)$ & $0.09(0.91,0.91)$ & $0.10(0.95,0.95)$ \\
Seoul & $-0.01(-0.06)$ & $-0.04(-0.33,-0.33)$ & $-0.01(-0.06,-0.06)$ \\
Work & $-0.18(-1.61)$ & $-0.25(-2.09,-2.11)$ & $-0.19(-1.64,-1.64)$ \\
kid-par & $0.03(1.81)$ & $0.02(1.47,1.48)$ & $0.03(1.83,1.83)$ \\
$\hat{v}_{2}$ & & $6.13(2.18,2.30)$ & $-0.01(-0.02,-0.03)$ \\
$\hat{v}_{2}^{2}$ & & $0.00(0.06,0.06)$ & $0.00(-0.16,-0.23)$ \\
$\hat{v}_{2} \times$ age & & $-0.07(-2.22,-2.33)$ & $0.00(-0.01,-0.01)$ \\
$\hat{v}_{2} \times$ male & & $-0.41(-1.11,-1.11)$ & $0.01(0.19,0.20)$ \\
$\hat{v}_{2} \times$ married & $0.29(1.36,1.31)$ & $0.02(0.53,0.56)$ \\
\hline & & &
\end{tabular}




\section{CONCLUSIONS}

This paper examined whether informal health care can reduce formal health care, where the informal care $y_{2}$ is the number of family caregivers ( $85 \%$ zeros) and the formal care $y_{1}$ is medical and long-term care expenditures (14\% zeros). This task posed a number of difficulties because $y_{2}$ is an endogenous regressor that is a count variable with too many zeros in addition to $y_{1}$ having a nontrivial proportion of zeros.

Facing the difficulties, we proposed a two-stage procedure where the first stage is estimating $E\left(y_{2} \mid x\right)$ as the product of logit (using $y_{2}$ being positive or not) and an exponential regression function (using only positive $y_{2}$ ) — the idea borrowed from 'zero-inflated Poisson'. The second stage is applying a semiparametric censored model estimator for $y_{1}$ with the endogeneity of $y_{2}$ removed by a CF. Two types of CFs were considered: one based on the additive residual $y_{2}-E\left(y_{2} \mid x\right)$ and the other based on the multiplicative residual $\left\{y_{2} / E\left(y_{2} \mid x\right)\right\}-1$; the actual CFs used were polynomial functions of these residuals and their interaction terms with regressors.

Despite the intuitive appeal of the multiplicative residual as an exponential function appears, the additive residual $\mathrm{CF}$ performed much better than the multiplicative residual CF. Also, using only an exponential function for $E\left(y_{2} \mid x\right)$ (i.e., ignoring the too many zero problem) was tried, but the outcome was inferior to the procedure with both logit and exponential functions. One important lesson learned in using CF was that, instead of using a single residual, its polynomial functions and interaction terms with regressors should be explored.

Our empirical result using Korean data for the elderly of age 65 years and above showed that informal care is a substitute for formal care only in certain cases such as people with diabetes (i.e., informal care interacts with diabetes), and there are weak evidences that informal care also interacts with high blood pressure, mental diseases, age, and male. That is, as noted in the literature of informal and formal care trade-off, the effect of informal care on formal care is heterogeneous.

In terms of policy implications, the government may promote more informal family care, for instance, by providing financial incentives for family members to stay home to take care of their parents, which can reduce the demand for formal care. The resulting reduction in formal care expense may be greater than the informal care promotion expenses. For instance, in Korea, the provisions to pay for informal home cares in the Korean long-term care support insurance may be expanded to promote further informal home cares.

\section{ACKNOWLEDGEMENTS}

The authors are grateful to two anonymous reviewers and Kamhon Kan for their detailed comments. Myoung-jae Lee's work was supported by the National Research Foundation of Korea grantfunded by the Korean Government (KRF-2009-342-B0008 and NRF-2011-327-B00072).

\section{REFERENCES}

Almirall D, Ten Have T, Murphy SA. 2010. Structural nested mean models for assessing time-varying effect moderation. Biometrics 66: 131-139.

Bolin K, Lindgren B, Lundborg P. 2008. Informal and formal care among single-living elderly in Europe. Health Economics 17: 393-409.

Bonsang E. 2009. Does informal care from children to their elderly parents substitute for formal care in Europe? Journal of Health Economics 28: 143-154.

Charles K, Sevak P. 2005. Can family caregiving substitute for nursing home care? Journal of Health Economics 24: 1174-1190.

Honoré B. 1992. Trimmed LAD and LSE of truncated and censored regression models with fixed effects. Econometrica 60: $533-565$.

Huang F, Lee MJ. 2010. Dynamic treatment effect analysis of TV effects on child cognitive development. Journal of Applied Econometrics 25: 392-419.

Kang SJ, Lee MJ. 2003. Analysis of private transfers with panel fixed effect censored model estimator. Economics Letters 80: $233-237$. 
Kang CH, Lee MJ. 2010. Performance of various estimators for censored response models with endogenous regressors. Pacific Economic Review 15: 474-490.

Lambert D. 1992. Zero-inflated Poisson regression, with an application to defects in manufacturing. Technometrics 34: 1-14. Lee MJ. 1992. Winsorized mean estimator for censored regression model. Econometric Theory 8: 368-382.

Lee MJ. 2010. Micro-Econometrics: Methods of Moments and Limited Dependent Variables. Springer.

Lee MJ. 2012a. Semiparametric estimators for limited dependent variable (LDV) models with endogenous regressors. Econometric Reviews 31: 171-214.

Lee MJ. 2012b. Treatment effects in sample selection models and their nonparametric estimation. Journal of Econometrics 167: $317-329$.

Lee MJ, Huang F. 2012. Finding dynamic treatment effects under anticipation: the effects of spanking on behavior. Journal of the Royal Statistical Society (Series A) 175: 535-567.

Levitt SD. 1996. How do senators vote? Disentangling the role of voter preferences, party affiliation, and senator ideology. American Economic Review 86: 425-441.

Mullahy J. 1986. Specification and testing of some modified count data models. Journal of Econometrics 33: 341-365.

Powell JL. 1984. Least absolute deviations estimation for the censored regression model. Journal of Econometrics 25: 303-325.

Powell JL. 1986. Symmetrically trimmed least squares estimation for Tobit models. Econometrica 54: 1435-1460.

Santos-Silva JMC. 2001. Influence diagnostics and estimation algorithms for Powell's SCLS. Journal of Business and Economic Statistics 19: 55-62.

Santos-Silva JMC, Tenreyro S. 2006. The log of gravity. The Review of Economics and Statistics 88: 641-658.

Terza JV, Basu A, Rathouz PJ. 2008. Two-stage residual inclusion estimation: addressing endogeneity in health econometric modeling. Journal of Health Economics 27: 531-543.

Van Houtven CH, Norton EC. 2004. Informal care and health care use of old adults. Journal of Health Economics 23: $1159-1180$. 